\title{
MAGNETIC ANISOTROPY AND MAGNETOSTRICTION OSCILLATIONS IN MAGNETIC MULTILAYERS
}

\author{
H. SzYMCZAK ${ }^{a *}$, R. ŻUBer.eK ${ }^{a}$ AND F. STOBIECKI ${ }^{b}$ \\ anstitute of Physics, Polish Academy of Sciences \\ Al. Lotników 32/46, 02-668 Warsaw, Poland \\ ${ }^{b}$ Institute of Molecular Physics. Polish Academy of Sciences \\ Smoluchowskiego 17, 60-179 Poznani, Poland
}

(Received April 1, 1999)

\begin{abstract}
Interlayes: pseudodtipolas: coupling between two ferromagnets separated by nommagnetic spacer is analyzed. It was shown that this type of interaction should induce magnetic anisotropy and magnetostriction oscillations. It was also predicted that both magnetic anisotropy and magnetostriction decrease with an increase in the spacer thickness. This effect was observed in $[\text { permalloy/Cu}]_{100}$ multilayers.
\end{abstract}

PACS numbers: 74.25.Ha, 75.70.-i. 75.70.Cn, 75.80.+q

\section{Introduction}

In recent years there has been a growing interest in stuclying the interlayer exchange coupling between ferromagnetic layers across non-magnetic layers. The predictions by Bruno and Chapper. [1] concerning the oscillatory behavior of the interlayer coupling have been successfully verified for $\mathrm{Co} / \mathrm{Cu}$ system by Johnson et al. [2]. The theoretical calculations have been performed within the frame of Ruderman-Kittel-Kasuya-Yoshida (RKKY) theory. 'The spin-orbit interaction is usually neglected in this type of calculations. Nevertheless, the broken (uniaxial) symmetry of the system under consideration and the spin-orbit interaction are expected to induce the anisotropic interlayer exchange coupling. Recently it has been shown that apart from the isotropic Heisenberg type exchange term, the exchange coupling between two magnetic impurities in a host metal also contains a Dzialoshinsky-Moriya term and a pseudodipolar term [3]. The Dzialoshinsky-Moriya term is responsible [4] for the non-collinear alignments among neighboring ferromagnetic layers described usually by a phenomenological biquadratic term $J\left(M_{1} \cdot M_{2}\right)^{2}$. The existence of the pseudodipolar term in RKKY interactions has been analyzed in the case of rare-earth ions by Irkhin and

\footnotetext{
*e-mail:szymh@ifpan.edu.pl
} 
Karpenko [5]. This type of the RKKY exchange interactions has been used to explain the origin of the perpendicular anisotropy and magnetostriction in amorphous Gd-Co films [6].

In this paper the consequences of the pseudodipolar RKKY interactions will be analyzed in application to the anisotropy and magnetostriction of the magnetic multilayer systems.

\section{Phenomenological model}

The exact analysis of the interlayer coupling requires the full knowledge of the band structure. For most of the analyzed systems such information is not available. Therefore, we limit our consideration to the phenomenological approach. Following the procedure developed in [4] it can be shown that the interlayer interaction resulting from the broken symmetry of the system and the spin-orbit interaction has the pseudodipolar term (apart from the isotropic one):

$$
H_{\mathrm{dip}}=\sum_{n, m} J_{d}(z)\left[M_{n}^{z} M_{m}^{z}-(1 / 3) \bar{M}_{n} \cdot \bar{M}_{m}\right],
$$

where $z$ is the spacer layer thickness and $M_{n}$ is the magnetization of the $n$-th ferromagnetic layer. $(m, n)$ implies a summation over pairs of nearest-neighbor: ferromagnetic layers. In a large spacer layer thickness approximation

$$
J_{d}(z) \sim \frac{\sin \left(2 k_{\mathrm{F}} z\right)}{z^{2}},
$$

where $k_{\mathrm{F}}$ is the Fermi vector.

The pseudodipolar interlayer interactions described by Eq. (1) give contribution to the magnelic anisotropy $K_{u}$ of the magnetic multilayers. The second order anisotropy coustant $K_{u}$ is proportional to

$$
K_{u} \sim M^{2} \frac{\sin \left(2 k_{\mathrm{F}} z\right)}{z^{2}} .
$$

Equation (3) predicts the magnetic anisotropy oscillations in magnetic multilayers. Such effect has not been observed ex] erimentally yet. Recently the magnetic anisotropy oscillations have been observed in Co films grown on $\mathrm{Cu}[7]$. The oscillations appear when $\mathrm{Cu}$ thickness is cluanged and are attributed to the periodic variations of the film morphology alternating between filled and incompletely filled atomic layers. Another type of oscillations have been observed for the same system for Co films with Cu overlayers. In this case the anisotropy of the Co film oscillates with Cu layer thickness. This effect is related to quantum interferences due to the confinement of electrons in ultrathin films [7]. The discovery of the magnetic anisotropy oscillations as a function of the Cu overlayer thickness seems to confirm expectation that the oscillations predicted by Eq. (3) should also be observed in magnetic multilayers.

Since magnetostriction arises from a dependence of the magnetic anisotropy energy on the state of strain of the lattice one should expect to observe an oscillation of magnetostriction constant $\lambda$ in magnetic multilayers. It can be shown that 


$$
\lambda \sim M^{2}\left[\frac{\sin \left(2 k_{\mathrm{F}} \approx\right)}{\dot{\sim}^{2}}+\frac{2 k_{\mathrm{F}} \cos \left(2 k_{\mathrm{F}} \dot{*}\right)}{\approx}\right] .
$$

This type of oscillations has never been observed in experiments. Equations (3) and (4) predict a very short oscillation period $\pi / k_{\mathrm{F}}$. Such period is almost impossible to detect experimentally because both magnetic anisotropy and magnetostriction should oscillate with a period of 1 monolayer. Such types of oscillations are attributed to the periodic variations of the film morphology alternating between filled and incompletely filled atomic layers.

Generally one should also expect the occurrence of long periods of oscillations. For $z>\pi / k_{\mathrm{F}}$ one should replace $2 k \mathrm{k}$ with $2 k \mathrm{~F}-G$, where $G$ is the translational vector belonging to the two-dimensional reciprocal lattice. The periods of these oscillations are cletermined by $G$ vector namely

$$
\frac{\pi}{\left[\left(2 k_{\mathrm{F}}\right)^{2}-\left(\mathrm{G}^{2}\right]^{1 / 2}\right.} \text {. }
$$

This result is quite general and allow us. in principle, to predict the oscillation periods for any spacer metal, knowing its Fermi surface. One should also take into account that structura. imperfections like misfit dislocations, interfacial roughness and strain have some effects on the Fermi surface and consequently on the periods of oscillations.

It seems that more detailed experimental studies are needed to observe the oscillations of magnetic anisotropy and magnetostriction in magnetic multilayers. The main purpose of this paper is to check experimentally the predictions expressed by Eqs. (4) and (5).

\section{Experimental results}

The experiment was performed on [permalloy/Cu] 100 multilayers. The samples were prepared by double face-to-face sputtering method [8] on glass substrates. The thickness of permalloy layer was almost constant equal to $2 \mathrm{~mm}$ and the thickness of Cu layer varied from $1.4 \mathrm{~nm}$ to $2.5 \mathrm{~nm}$. The thicknesses were later controlled by the analysis of low diffraction scans and X-ray fluorescence method [9].

The magnetostriction constants were measured at room temperature by the strain-modulated ferromagnetic resonance (SMFMR) method [10] at $9.10 \mathrm{GHz}$ with an external magnetic field and uniaxial stress parallel to the film plane and perpendicular to each other. In the SMFMR technique the strain, periodic in time, causes a modulation of the ferromagnetic resonance (FMR) line position. To obtain the values of the components of the magnetoelastic tensor one should compare the intensity of the SMFMR line with the intensity of the same line detected using the standard FMR technique.

Figure 1 shows the dependence of the magnetostriction constant $\lambda_{s}$ on the thickness of Cu layer $\left(d_{C u}\right)$. Although the existence of the oscillations of magnetostriction constants is difficult to establish it is seen that $\lambda_{\mathrm{s}}$ decreases with $d_{\mathrm{Cu}}$ in accordance with theoretical predictions. It is the first experimental evidence of the effect of the nommagnetic spacer on the magnetostriction of magnetic multilayers. 


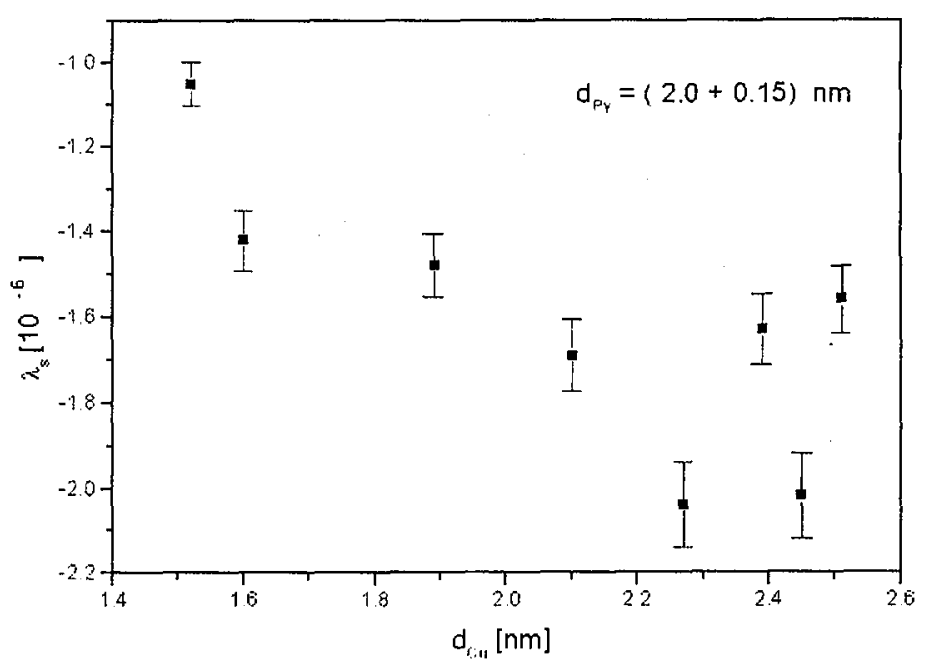

Fig. 1. The magnetostriction constant $\lambda_{s}$ of $[\text { permalloy/Cu }]_{100}$ multilayers versus $d_{\mathrm{Cu}}, d_{\text {permalloy }}=2 \mathrm{~nm}$.

\section{Conclusions}

In conclusions, oscillations of the uniaxial magnetic anisotropy and magnetostriction were predicted to exist in magnetic multilayers. These oscillations are related to the pseudodipolar RKKY interactions arising because of the broken symmetry of the system due to the spin -orbit interactions. To confirm the theoretical predictions magnetostriction of [permalloy/Cu$]_{100}$ multilayers has been studied as a function of nonmagnetic spacer thickness. Although the oscillations of magnetostriction constant were not shown to exist the decrease in the magnetostriction with the spacer thickness predicted by Eq. (4) was observed for the first time.

\section{Acknowledgments}

This work was supported by the Committee for Scientific Research under grant No. 2 P03B 03109.

\section{References}

[1] P. Bruno, C. Chappert, Phys. Rev. Lett. 67, 1602 (1991).

[2] M.T. Johnson, S.T. Purcell, N.W.E. McGee, R. Coehoorn, J. van de Stegge, W. Hoving, Phys. Rev. Lett. 68, 2688 (1992).

[3] J.B. Staunton, B.L. Gyorffy, J. Poulter, P. Strange, J. Phys. C 21, 1595 (1988).

[4] Ke Xia, Weiyi Zhang, Mu Lu, Hongru Zhai, Phys. Rev. B 55, 12561 (1997).

[5] Yu.P. Irkhin, V.P. Karpenko, Fiz. Tverd. Tela 13, 3586 (1971).

[6] K. Twarowski, H.K. Lachowicz, M. Gutowski, H. Szymczak, Phys. Status Solidi A 63, 103 (1981). 
[7] C.H. Back, W. Weber, Ch. Wursch, A. Bishof, D. Pescia, R. Allenspach, J. Appl. Phys. 81, 5054 (1997).

[8] F. Stobiecki, J. Dubowik, T. Luciński, B. Szymański, H. Rohrmann, K. Roll, M. Schmidt. Acta Phys. Pol. A 91. 277 (1997).

[9] J. Baszyński, F. Stobiecki, B. Szymański, K. Chrzumnicka, Phys. Status Solidi A 141, K23 (1994).

[10] H. Szymcza.k, J. Wosik, W. Zbieranowski, A. Dąbkowski, in: Proc. 4th Int. Conf. Microwave Ferrites, Jabtonna (Poland) 1978, Institute of Physics, Polish Academy of Sciences, Warsa.w 1978, p. 247. 